\title{
On Asymptotic Behavior of Solutions of Generalized Emden-Fowler Differential Equations with Delay Argument
}

\author{
Alexander Domoshnitsky ${ }^{1}$ and Roman Koplatadze ${ }^{2}$ \\ ${ }^{1}$ Department of Mathematics and Computer Sciences, Ariel University, 40700 Ariel, Israel \\ ${ }^{2}$ Department of Mathematics, Tbilisi State University, University Street 2, 0143 Tbilisi, Georgia
}

Correspondence should be addressed to Alexander Domoshnitsky; adom@ariel.ac.il

Received 4 July 2013; Accepted 8 November 2013; Published 22 January 2014

Academic Editor: Josef Diblik

Copyright (c) 2014 A. Domoshnitsky and R. Koplatadze. This is an open access article distributed under the Creative Commons Attribution License, which permits unrestricted use, distribution, and reproduction in any medium, provided the original work is properly cited.

\begin{abstract}
The following differential equation $u^{(n)}(t)+p(t)|u(\sigma(t))|^{\mu(t)}$ sign $u(\sigma(t))=0$ is considered. Here $p \in L_{\text {loc }}\left(R_{+} ; R_{+}\right), \mu \in$ $C\left(R_{+} ;(0,+\infty)\right), \sigma \in C\left(R_{+} ; R_{+}\right), \sigma(t) \leq t$, and $\lim _{t \rightarrow+\infty} \sigma(t)=+\infty$. We say that the equation is almost linear if the condition $\lim _{t \rightarrow+\infty} \mu(t)=1$ is fulfilled, while if $\limsup _{t \rightarrow+\infty} \mu(t) \neq 1$ or $\liminf _{t \rightarrow+\infty} \mu(t) \neq 1$, then the equation is an essentially nonlinear differential equation. In the case of almost linear and essentially nonlinear differential equations with advanced argument, oscillatory properties have been extensively studied, but there are no results on delay equations of this sort. In this paper, new sufficient conditions implying Property A for delay Emden-Fowler equations are obtained.
\end{abstract}

\section{Introduction}

This work deals with oscillatory properties of solutions of a functional differential equation of the form

$$
u^{(n)}(t)+p(t)|u(\sigma(t))|^{\mu(t)} \operatorname{sign} u(\sigma(t))=0,
$$

where

$$
\begin{array}{cc}
p \in L_{\mathrm{loc}}\left(R_{+} ; R\right), & \mu \in C\left(R_{+} ;(0 ;+\infty)\right), \\
\sigma \in C\left(R_{+} ; R_{+}\right), & \sigma(t) \leq t \quad \text { for } t \in R_{+}, \\
\lim _{t \rightarrow+\infty} \sigma(t)=+\infty . &
\end{array}
$$

It will always be assumed that the condition

$$
p(t) \geq 0 \quad \text { for } t \in R_{+}
$$

is fulfilled.

Let $t_{0} \in R_{+}$. A function $u:\left[t_{0} ;+\infty\right) \rightarrow R$ is said to be a proper solution of (1) if it is locally absolutely continuous together with its derivatives up to order $n-1$ inclusive,

$$
\sup \{|u(s)|: s \in[t ;+\infty)\}>0 \text { for } t \geq t_{0}
$$

and there exists a function $\bar{u} \in C\left(R_{+} ; R\right)$ such that $\bar{u}(t) \equiv u(t)$ on $\left[t_{0} ;+\infty\right)$ and the equality $\bar{u}^{(n)}(t)+$ $p(t)|\bar{u}(\sigma(t))|^{\mu(t)}$ sign $\bar{u}(\sigma(t))=0$ holds for $t \in\left[t_{0}:+\infty\right)$. A proper solution $u:\left[t_{0}:+\infty\right) \rightarrow R$ of (1) is said to be oscillatory if it has a sequence of zeros tending to $+\infty$. Otherwise the solution $u$ is said to be nonoscillatory.

Definition 1. We say that (1) has Property A if any of its proper solutions is oscillatory when $n$ is even and either is oscillatory or satisfies

$$
\left|u^{(i)}(t)\right| \downarrow 0 \quad \text { for } t \uparrow+\infty \quad(i=0, \ldots, n-1),
$$

when $n$ is odd.

Definition 2. We say that (1) is almost linear if the condition $\lim _{t \rightarrow+\infty} \mu(t)=1$ holds, while if $\limsup _{t \rightarrow+\infty} \mu(t) \neq 1$ or $\liminf \operatorname{int}_{t \rightarrow+\infty} \mu(t) \neq 1$, then we say that the equation is an essentially nonlinear differential equation.

The Emden-Fowler equation originated from theories concerning gaseous dynamics in astrophysics in the middle of the nineteenth century. In the study of stellar structure at 
that time it was important to investigate the equilibrium configuration of the mass of spherical clouds of gas. Lord Kelvin in 1862 assumed that the gaseous cloud is under convective equilibrium and then Lane [1] studied the equation

$$
\frac{1}{t^{2}} \frac{d}{d t}\left(t^{2} \frac{d u}{d t}\right)+u^{\gamma}=0 .
$$

The Emden-Fowler equations were first considered only for second-order equations and written in the form

$$
\frac{d}{d t}\left(p(t) \frac{d u}{d t}\right)+q(t) u^{\gamma}=0, \quad t \geq 0,
$$

which could be reduced in the case of positive and continuous coefficients to the equation

$$
x^{\prime \prime}+a(t) x^{\gamma}=0, \quad t \geq 0
$$

To avoid difficulties of defining $x^{\gamma}$ when $x(t)$ is negative and $\gamma$ is not an integer, the equation

$$
x^{\prime \prime}(t)+a(t)|x(t)|^{\gamma} \operatorname{sign} x(t)=0, \quad t \geq 0,
$$

was usually considered. The mathematical foundation of the theory of such equations was built by Fowler [2] and the description of the results can be found in Chapter 7 of [3].

We see also the Emden-Fowler equation in gas dynamics and fluid mechanics (see Sansone [4], page 431 and the paper [5]). Nonoscillation of these equations is important in various applications. Note that the zero of such solutions corresponds to an equilibrium state in a fluid with spherical distribution of density and under mutual attraction of its particles. The Emden-Fowler equations can be either oscillatory (i.e., all proper solutions have a sequence of zeros tending to zero) or nonoscillatory, if solutions are eventually positive or negative, or, in contrast with the case of linear differential equations of second order, may possess both oscillating and nonoscillating solutions. For example, for the equation

$$
x^{\prime \prime}(t)+t^{\mu}|x(t)|^{\gamma} \operatorname{sign} x(t)=0, \quad t \geq 0,
$$

it was proven in [2] that for $\mu \geq-2>-(\gamma+3) / 2$ all solutions oscillate, for $\mu<-(\gamma+3) / 2$-all solutions nonoscillate, and for $-(\gamma+3) / 2 \leq \mu<-2$ there are both oscillating and nonoscillating solutions.

The Emden-Fowler equation presents one of the classical objects in the theory of differential equations. Tests for oscillation and nonoscillation of all solutions and existence of oscillating solutions were obtained in the works [6-8]. In [9] for the case $0<\gamma<1$, it was obtained that all solutions of the equation

$$
x^{\prime \prime}(t)+a(t)\left|x^{\gamma}(t)\right| \operatorname{sign} x(t)=0, \quad t \geq 0,
$$

oscillate if and only if

$$
\int_{0}^{\infty} t^{\gamma} a(t) d t=\infty
$$

The latest research results in this area are presented in the book [8]. Behavior of solutions to $n$th order Emden-Fowler equations can be essentially more complicated. Properties A and B defined by Kiguradze are studied in the abovementioned book.

There are essentially less results on oscillation of delay Emden-Fowler equations. Oscillation properties of nonlinear delay differential equations, where Emden-Fowler equations were also included as a particular case, were studied in [10$20]$. Results of these papers are discussed in $[13,15]$, where various examples demonstrating essentialities of conditions are also presented. Note that for delay differential equations there are no results on nonoscillation of all solutions and only existence of nonoscillating solutions is studied. Actually, the results on oscillation of delayed equations are based on the approaches existing for ordinary differential equations with development in the direction of preventing the obstructive influence of delay. In the paper [15] the following equation

$$
x^{(n)}(t)+a(t) f[x(\sigma(t))]=0, \quad t \geq 0, n \text { even, }
$$

and its particular case

$$
\begin{array}{r}
x^{(n)}(t)+a(t)|x(\sigma(t))|^{\gamma} \operatorname{sign} x(\sigma(t))=0, \\
t \geq 0, n \text { even, }
\end{array}
$$

are considered. It was obtained for the last equation under some standard assumptions on the coefficients [15] that in the case $0<\gamma<1$,

$$
\int_{0}^{\infty} \sigma^{(n-1) \gamma}(t) a(t) d t=\infty
$$

all solutions oscillate. We see that the integral depends on deviation of argument $\sigma(t)$ and the power of the equation $n$. For the equation

$$
\begin{array}{r}
x^{(n)}(t)+a(t) \prod_{i=1}^{m}\left|x\left(\sigma_{i}(t)\right)\right|^{\gamma_{i}} \operatorname{sign} x\left(\sigma_{i}(t)\right)=0, \\
t \geq 0, \quad n \text { even, }
\end{array}
$$

where $\gamma_{i}$ is the ratio of two positive odd integers, $\sigma(t) \leq$ $\sigma_{i}(t) \leq t$ for $i=1, \ldots, m$, and $\sigma(t) \rightarrow \infty$ as $t \rightarrow \infty$, each of the following conditions (a), (b), and (c) ensures oscillation of all solutions:

(a)

$$
\int_{0}^{\infty} \sigma^{(n-1) \gamma}(t) a(t) d t=\infty \quad \text { for } \gamma=\sum_{i=1}^{m} \gamma_{i}<1 ;
$$

(b)

$$
\int_{0}^{\infty} \sigma^{(n-1) \alpha}(t) a(t) d t=\infty \text { for } \gamma=1,0<\alpha<1
$$

(c)

$$
\int_{0}^{\infty} \sigma^{n-1}(t) a(t) d t=\infty, \quad \sigma^{\prime}(t) \geq 0 \quad \text { for } \gamma>1 .
$$


Most proofs of results on oscillation of all solutions to second order equations utilize the fact that if a nonoscillating solution exists, the signs of the solution $x(t)$ and its second derivative $x^{\prime \prime}(t)$ are opposite to each other for sufficiently large $t$. Then a growth of nonoscillating solution is estimated and the authors come to contradiction with conditions that proves oscillation of all solutions. Note that delays disturb oscillation. Instead of $t^{\gamma}, \sigma^{\gamma}(t)$ appears. The principle is clear: for oscillation of all solutions we have to achieve a corresponding smallness of the delay $t-\sigma(t)$. All this is more complicated if we study $n$th order equations. In this case also the fact that $x(t)$ and its $n$th derivative $x^{(n)}(t)$ have different signs for sufficiently large $t$ is used, but the technique is more complicated.

In the papers [21-28] a generalization of Emden-Fowler equations was considered. The powers in these papers can be functions and not constants. In many cases, it leads to essentially new oscillation properties of such equations. Surprisingly, oscillation behavior of equations, with the power $\lambda$ and with functional power $\mu(t)$ such that $\lim _{t \rightarrow \infty} \mu(t)=$ $\lambda$, can be quite different. The main purpose of our paper is to study conditions under which the generalized (in this sense) equations preserve the known oscillation properties of Emden-Fowler equations and conditions under which these properties are not preserved. Oscillatory properties of almost linear and essentially nonlinear differential equation with advanced argument have already been studied in [21-28]. In this paper we study oscillation properties of $n$th order delay Emden-Fowler equations.

\section{Some Auxiliary Lemmas}

In the sequel, $\widetilde{C}_{\text {loc }}\left(\left[t_{0} ;+\infty\right)\right)$ will denote the set of all functions $u:\left[t_{0} ;+\infty\right) \rightarrow R$ absolutely continuous on any finite subinternal of $\left[t_{0} ;+\infty\right)$ along with their derivatives of order up to including $n-1$.

Lemma 3 (see [28]). Let $u \in \widetilde{C}_{\text {loc }}^{n-1}\left(\left[t_{0} ;+\infty\right)\right), u(t)>0$, $u^{(n)}(t) \leq 0$ for $t \geq t_{0}$, and $u^{(n)}(t) \quad \equiv \quad 0$ in any neighborhood of $+\infty$. Then there exist $t_{1} \geq t_{0}$ and $\ell \in\{0, \ldots, n-1\}$ such that $\ell+n$ is odd and

$$
\begin{gathered}
u^{(i)}(t)>0 \quad \text { for } t \geq t_{1}(i=0, \ldots, \ell-1), \\
(-1)^{i+\ell} u^{(i)}(t)>0 \text { for } t \geq t_{1}(i=\ell, \ldots, n-1) .
\end{gathered}
$$

Remark 4. If $n$ is odd and $\ell=0$, then it means that in $\left(20_{0}\right)$ only the second inequalities are fulfilled.

Lemma 5 (see [29]). Let $u \in \widetilde{C}_{\mathrm{loc}}^{n-1}\left(\left[t_{0} ;+\infty\right)\right)$ and let $\left(20_{\ell}\right)$ be fulfilled for some $\ell \in\{0, \ldots, n-1\}$ with $\ell+n$ odd. Then

$$
\int_{t_{0}}^{+\infty} t^{n-\ell-1}\left|u^{(n)}(t)\right| d t<+\infty .
$$

If, moreover,

$$
\int_{t_{0}}^{+\infty} t^{n-\ell}\left|u^{(n)}(t)\right| d t=+\infty
$$

then there exists $t_{*}>t_{0}$ such that

$$
\begin{gathered}
\frac{u^{(i)}(t)}{t^{\ell-i}} \downarrow, \quad \frac{u^{(i)}(t)}{t^{\ell-i-1}} \uparrow \quad(i=0, \ldots, \ell-1), \\
u(t) \geq \frac{t^{\ell-1}}{\ell !} u^{(\ell-1)}(t) \quad \text { for } t \geq t_{*}, \\
u^{(\ell-1)}(t) \geq \frac{t}{(n-\ell) !} \int_{t}^{+\infty} s^{n-\ell-1}\left|u^{(n)}(s)\right| d s \\
+\frac{1}{(n-\ell) !} \int_{t_{*}}^{t} s^{n-\ell}\left|u^{(n)}(s)\right| d s \quad \text { for } t \geq t_{*} .
\end{gathered}
$$

\section{Necessary Conditions for the Existence of a Solution of Type $\left(20_{\ell}\right)$}

The following notation will be used throughout the work:

$$
\begin{gathered}
\alpha=\inf \left\{\mu(t): t \in R_{+}\right\}, \quad \beta=\sup \left\{\mu(t): t \in R_{+}\right\}, \\
\sigma_{(-1)}(t)=\sup \{s \geq 0, \sigma(s) \leq t\}, \\
\sigma_{(-k)}=\sigma_{(-1)} \circ \sigma_{(-(k-1))}, \quad k=2,3, \ldots
\end{gathered}
$$

Clearly $\sigma_{(-1)}(t) \geq t$, and $\sigma_{(-1)}$ is nondecreasing and coincides with the inverse of $\sigma$ when the latter exists.

Definition 6. Let $t_{0} \in R_{+}$. By $\mathbf{U}_{\ell, t_{0}}$ one denotes the set of all proper solutions $u:\left[t_{0},+\infty\right) \rightarrow R$ of (1) satisfying the condition $\left(20_{\ell}\right)$ with some $t_{1} \geq t_{0}$.

Lemma 7. Let the conditions (2), (3) be fulfilled, let $\ell \in$ $\{1, \ldots, n-1\}$ with $\ell+n$ odd, and let $u \in \mathbf{U}_{\ell, t_{0}}$ be a positive proper solution of (1). If, moreover, $\alpha \geq 1, \beta<+\infty$,

$$
\int_{0}^{+\infty} t^{n-\ell}(\sigma(t))^{(\ell-1) \mu(t)} p(t) d t=+\infty,
$$

then for any $M \in(1 ;+\infty)$ there exists $t_{*}>t_{0}$ such that for any $k \in N$ 
where $\alpha$ is given by the first equality of (26) and

$$
\begin{aligned}
& \rho_{1, \ell, t_{*}}^{(\alpha)}(t) \\
& =\ell ! \exp \left\{M_{\ell}(\alpha) \int_{\sigma_{(-1)}\left(t_{*}\right)}^{t} \int_{s}^{+\infty} \xi^{n-\ell-2}\right. \\
& \times(\sigma(\xi))^{1+(\ell-1) \mu(\xi)} \\
& \times p(\xi) d \xi d s\}, \\
& \rho_{i, \ell, t_{*}}^{(\alpha)}(t)=\ell ! \\
& +\frac{1}{(n-\ell) !} \\
& \times \int_{\sigma_{(-i)}\left(t_{*}\right)}^{t} \int_{s}^{+\infty} \xi^{n-\ell-1}(\sigma(\xi))^{(\ell-1) \mu(\xi)} \times p(\xi) \\
& \times\left(\frac{1}{\ell !} \rho_{i-1, \ell, t_{*}}(\sigma(\xi))\right)^{\mu(\xi)} d \xi d s \\
& (i=2, \ldots, k), \\
& M_{\ell}(\alpha)= \begin{cases}\frac{1}{\ell !(n-\ell) !} & \text { if } \alpha=1, \\
M & \text { if } \alpha>1 .\end{cases}
\end{aligned}
$$

Proof. Let $t_{0} \in R_{+}, \ell \in\{1, \ldots, n-1\}$ with $\ell+n$ odd and $u \in \mathbf{U}_{\ell, t_{0}}$ (see Definition 6) is solution of (1). Since $\beta<+\infty$, according to $(1),\left(20_{\ell}\right)$, and $\left(28_{\ell}\right)$, it is clear that condition (22) holds. Thus, by Lemma 5 there exists $t_{2}>t_{1}$ such that the conditions $\left(23_{i}\right)-(25)$ with $t_{*}=t_{2}$ are fulfilled and

$$
\begin{aligned}
u^{(\ell-1)}(t) \geq & \frac{t}{(n-\ell) !} \int_{t}^{+\infty} s^{n-\ell-1} p(s)(u(\sigma(s)))^{\mu(s)} d s \\
& +\frac{1}{(n-\ell) !} \int_{t_{2}}^{t} s^{n-\ell} p(s)(u(\sigma(s)))^{\mu(s)} d s
\end{aligned}
$$

$$
\text { for } t \geq t_{2} \text {. }
$$

Observe that there exists $t_{3}>t_{2}$ such that $\sigma(t) \geq t_{2}$ for $t \geq t_{3}$. Thus, by (24), for any $t \geq t_{3}$ we get

$$
\begin{aligned}
& u^{(\ell-1)}(t) \\
& \geq \frac{t}{(n-\ell) !} \int_{t}^{+\infty} s^{n-\ell-1} p(s)(u(\sigma(s)))^{\mu(s)} d s \\
& \quad-\frac{1}{(n-\ell) !} \int_{t_{2}}^{t} s d \int_{s}^{+\infty} \xi^{n-\ell-1} p(\xi) \\
& \quad \times(u(\sigma(\xi)))^{\mu(\xi)} d \xi d s
\end{aligned}
$$

$$
\begin{aligned}
\geq \frac{1}{(n-\ell) !} \int_{t_{3}}^{t} \int_{s}^{+\infty} & \xi^{n-\ell-1}(\sigma(\xi))^{(\ell-1) \mu(\xi)} \\
& \times\left(\frac{1}{\ell !} u^{(\ell-1)}(\sigma(\xi))\right)^{\mu(\xi)} p(\xi) d \xi d s .
\end{aligned}
$$

According to $\left(28_{\ell}\right)$ and $\left(23_{\ell-1}\right)$, choose $t_{*}>t_{3}$ such that

$$
\begin{aligned}
\frac{1}{(n-\ell) !} \int_{t_{3}}^{t_{*}} \int_{s}^{+\infty} & \xi^{n-\ell-1}(\sigma(\xi))^{(\ell-1) \mu(\xi)} \\
& \times\left(\frac{u^{(\ell-1)}(\sigma(\xi))}{\ell !}\right)^{\mu(\xi)} p(\xi) d \xi d s>\ell ! .
\end{aligned}
$$

By (34) and (35) we have

$$
\begin{aligned}
& u^{(\ell-1)}(t) \geq \ell ! \\
& +\frac{1}{(n-\ell) !} \int_{t_{*}}^{t} \int_{s}^{+\infty} \xi^{n-\ell-1}(\sigma(\xi))^{(\ell-1) \mu(\xi)} p(\xi) \\
& \times\left(\frac{u^{(\ell-1)}(\sigma(\xi))}{\ell !}\right)^{\mu(\xi)} d \xi d s \\
& \text { for } t \geq t_{*} .
\end{aligned}
$$

Let $\alpha=1$. Since $u^{(\ell-1)}(t) \rightarrow+\infty$ as $t \rightarrow+\infty$, without loss of generally we can assume that $u^{(\ell-1)}(\sigma(\xi)) \geq \ell$ ! for $\xi \geq t_{3}$. Then by $\left(23_{\ell}\right)$ from $(36)$ we get

$$
\begin{aligned}
& u^{(\ell-1)}(t) \\
& \geq \ell !+\frac{1}{\ell !(n-\ell) !} \int_{t_{*}}^{t} \int_{s}^{+\infty} \xi^{n-\ell-2} \\
& \\
& \quad \times(\sigma(\xi))^{1+(\ell-1) \mu(\xi)} u^{(\ell-1)}(\xi) p(\xi) d \xi d s \\
& \quad \text { for } t \geq t_{*} .
\end{aligned}
$$

It is obvious that

$$
x^{\prime}(t) \geq \frac{u^{(\ell-1)}(t)}{\ell !(n-\ell) !} \int_{t}^{+\infty} \xi^{n-\ell-2}(\sigma(\xi))^{1+(\ell-1) \mu(\xi)} p(\xi) d \xi
$$

where

$$
\begin{aligned}
x(t)= & \ell !+\frac{1}{\ell !(n-\ell) !} \int_{t_{*}}^{t} \int_{s}^{+\infty} \xi^{n-\ell-2} \\
& \times(\sigma(\xi))^{1+(\ell-1) \mu(\xi)} p(\xi) u^{(\ell-1)}(\xi) d \xi d s .
\end{aligned}
$$


Thus, according to $\left(23_{\ell-1}\right)$ (37), and (39) from (38) we get

$$
\begin{aligned}
& x^{\prime}(t) \\
& \quad \geq \frac{x(t)}{\ell !(n-\ell) !} \int_{t}^{+\infty} \xi^{n-\ell-2}(\sigma(\xi))^{1+(\ell-1) \mu(\xi)} p(\xi) d \xi
\end{aligned}
$$$$
\text { for } t \geq t_{*} \text {. }
$$

Therefore

$x(t)$

$$
\begin{aligned}
\geq \ell ! \exp \left\{\frac{1}{\ell !(n-\ell) !}\right. \\
\left.\quad \times \int_{t_{*}}^{t} \int_{s}^{+\infty} \xi^{n-\ell-2}(\sigma(\xi))^{1+(\ell-1) \mu(\xi)} p(\xi) d \xi d s\right\}
\end{aligned}
$$$$
\text { for } t \geq t_{*} \text {. }
$$

Hence, according to (37) and (39)

$$
u^{(\ell-1)}(t) \geq \rho_{1, \ell, t_{*}}^{(1)}(t) \quad \text { for } t \geq \sigma_{(-1)}\left(t_{*}\right),
$$

where

$$
\begin{aligned}
& \rho_{1, \ell, t_{*}}^{(1)}(t) \\
& =\ell ! \exp \left\{\frac{1}{\ell !(n-\ell) !}\right. \\
& \quad \times \int_{\sigma_{(-1)}\left(t_{*}\right)}^{t} \int_{s}^{+\infty} \xi^{n-\ell-2} \\
& \left.\quad \times(\sigma(\xi))^{1+(\ell-1) \mu(\xi)} p(\xi) d \xi d s\right\} .
\end{aligned}
$$

Thus, according to (36) and (42)

$$
u^{(\ell-1)}(t) \geq \rho_{i, \ell, t_{*}}^{(1)}(t) \quad \text { for } t \geq \sigma_{(-i)}\left(t_{*}\right)(i=1, \ldots, k),
$$

where

$$
\begin{aligned}
& \rho_{i, \ell, t_{*}}^{(1)}(t) \\
& =\ell !+\frac{1}{(n-\ell) !} \\
& \quad \times \int_{\sigma_{(-i)}\left(t_{*}\right)}^{t} \int_{s}^{+\infty} \xi^{n-\ell-1}(\sigma(\xi))^{(\ell-1) \mu(\xi)} p(\xi) \\
& \times\left(\frac{1}{\ell !} \rho_{i-1, \ell, t_{*}}^{(1)}(\sigma(\xi))\right)^{\mu(\xi)} d \xi d s \\
& \quad(i=2, \ldots, k) .
\end{aligned}
$$

Now assume that $\alpha>1$ and $M \in(1,+\infty)$. Since $u^{(\ell-1)}(t) \uparrow$ $+\infty$ for $t \uparrow+\infty$, without loss of generality we can assume that $\left(u^{(\ell-1)}(\sigma(\xi)) / \ell !\right)^{\alpha-1} \geq \ell !(n-\ell) ! M$ for $\xi \geq t_{*}$. Therefore, from (36) we have

$$
\begin{aligned}
& u^{(\ell-1)}(t) \\
& \quad \geq \ell !+M \int_{t_{*}}^{t} \int_{s}^{+\infty} \xi^{n-\ell-1}(\sigma(\xi))^{(\ell-1) \mu(\xi)}
\end{aligned}
$$

$$
\times p(\xi) u^{(\ell-1)}(\sigma(\xi)) d \xi d s
$$

$$
\text { for } t \geq t_{*} \text {. }
$$

Taking into account (46), as above we can find that if $\alpha>1$, then

$$
u^{(\ell-1)}(t) \geq \rho_{k, \ell, t_{*}}^{(\alpha)}(t) \quad \text { for } t \geq \sigma_{(-k)}\left(t_{*}\right)
$$

where

$$
\begin{aligned}
& \rho_{1, \ell, t_{*}}^{(\alpha)}(t) \\
& \quad=\ell ! \exp \left\{M \int_{\sigma_{(-1)}\left(t_{*}\right)}^{t} \int_{s}^{+\infty} \xi^{n-\ell-2}(\sigma(\xi))^{1+(\ell-1) \mu(\xi)}\right.
\end{aligned}
$$$$
\times p(\xi) d \xi d s\}
$$$$
\text { for } t \geq \sigma_{(-1)}\left(t_{*}\right) \text {, }
$$

$$
\begin{aligned}
& \rho_{i, \ell, t_{*}}^{(\alpha)}(t)= \ell !+\frac{1}{(n-\ell) !} \\
& \times \int_{\sigma_{(-i)}\left(t_{*}\right)}^{t} \int_{s}^{+\infty} \xi^{n-\ell-1}(\sigma(\xi))^{(\ell-1) \mu(\xi)} p(\xi) \\
& \times\left(\frac{1}{\ell !} \rho_{i-1, \ell, t_{*}}^{(\alpha)}(\sigma(\xi))\right)^{\mu(\xi)} d \xi d s \\
& \quad \text { for } t \geq \sigma_{(-i)}\left(t_{*}\right)(i=2, \ldots, k) .
\end{aligned}
$$

According to (43)-(45) and (47)-(49), it is obvious that for any $\alpha \geq 1, k \in N$, and $M>1$ there exists $t_{*} \in R_{+}$such that (29)-(31) hold, where $M_{\ell}(\alpha)$ is defined by (32). This proves the validity of the lemma.

Analogously we can prove.

Lemma 8. Let conditions (2), (3), $\left(28_{\ell}\right)$ be fulfilled, let $\ell \in$ $\{1, \ldots, n-1\}$ with $\ell+n$ odd, $1 \leq \beta<+\infty$, and let $u \in \mathbf{U}_{\ell, t_{0}}$ be a positive proper solution of $(1)$. Then for any $M \in(1 ;+\infty)$ there exists $t_{*}>t_{0}$ such that for any $k \in N$

$$
u^{(\ell-1)}(t) \geq \tilde{\rho}_{k, \ell, t_{*}}^{(\beta)}(t) \quad \text { for } t \geq \sigma_{(-k)}\left(t_{*}\right)
$$


where $\beta$ is defined by the second equality of (26) and

$$
\begin{aligned}
\tilde{\rho}_{1, \ell, t_{*}}^{(\beta)}(t)= & \ell ! \exp \\
& \times\{M(\beta) \\
& \times \int_{\sigma_{(-1)}\left(t_{*}\right)}^{t} \int_{s}^{+\infty} \xi^{n-\ell-2} \times(\sigma(\xi))^{1+\ell \mu(\xi)-\beta} \\
& \times p(\xi) d \xi d s\},
\end{aligned}
$$

$$
\begin{aligned}
& \tilde{\rho}_{i, \ell, t_{*}}^{(\beta)}(t) \\
& =\ell !+\frac{1}{(n-\ell) !} \\
& \times \int_{\sigma_{(-i)}\left(t_{*}\right)}^{t} \int_{s}^{+\infty} \xi^{n-\ell-1}(\sigma(\xi))^{(\ell-1) \mu(\xi)} \\
& \times\left(\frac{1}{\ell !} \tilde{\rho}_{i-1, \ell, t_{*}}(\sigma(\xi))\right)^{\mu(\xi)} p(\xi) d \xi d s \\
& M(\beta)= \begin{cases}\frac{1}{\ell !(n-\ell) !} & \text { if } \beta=1, \\
M & \text { if } \beta>1 .\end{cases}
\end{aligned}
$$

Remark 9. In Lemma 7, the condition $\beta<+\infty$ cannot be replaced by the condition $\beta=+\infty$. Indeed, let $c \in(0,1)$. Consider (1), where $n$ is even and

$$
\begin{gathered}
\sigma(t) \equiv t, \quad p(t)=\frac{n ! t^{\log _{1 / c} t}}{t^{n+1}(c t-1)^{\log _{1 / c} t}}, \\
\beta(t)=\log _{1 / c} t, \quad t \geq \frac{2}{c} .
\end{gathered}
$$

It is obvious that the function $u(t)=c-(1 / t)$ is the solution of (1) and it satisfies the condition $\left(20_{1}\right)$ for $t \geq$ $(2 / c)$. On the other hand, the condition $\left(28_{1}\right)$ holds, but the condition (22) is not fulfilled.

Theorem 10. Let $\ell \in\{1, \ldots, n-1\}$ with $\ell+n$ be odd, let $\beta<$ $+\infty$ and the conditions (2), (3), (28 $)$, and let

$$
\int_{0}^{+\infty} t^{n-\ell-1}(\sigma(t))^{\ell \mu(t)} p(t) d t=+\infty
$$

be fulfilled, and for some $t_{0} \in R_{+}, \mathbf{U}_{\ell, t_{0}} \neq \emptyset$. Then for any $M>1$ there exists $t_{*}>t_{0}$ such that if $\alpha=1$,

$$
\begin{aligned}
\lim _{t \rightarrow+\infty} \frac{1}{t} \int_{\sigma_{(-k)}\left(t_{*}\right)}^{t} \int_{s}^{+\infty} \xi^{n-\ell-1}(\sigma(\xi))^{(\ell-1) \mu(\xi)} \\
\quad \times\left(\frac{1}{\ell !} \rho_{k, \ell, t_{*}}^{(1)}(\sigma(\xi))\right)^{\mu(\xi)} p(\xi) d \xi d s=0
\end{aligned}
$$

and if $\alpha>1$, then for any $k \in N$ and $\delta \in(1 ; \alpha]$,

$$
\begin{aligned}
\int_{\sigma_{(-k)}\left(t_{*}\right)}^{+\infty} \int_{s}^{+\infty} \xi^{n-\ell-1-\delta}(\sigma(\xi))^{(\ell-1) \mu(\xi)+\delta} \\
\quad \times\left(\frac{1}{\ell !} \rho_{k, \ell, t_{*}}^{(\alpha)}(\sigma(\xi))\right)^{\mu(\xi)-\delta} p(\xi) d \xi d s<+\infty,
\end{aligned}
$$

where $\alpha$ is defined by first equality of (26) and $\rho_{k, \ell, t_{*}}^{(\alpha)}$ is given by (30)-(32).

Proof. Let $M>1$ and $t_{0} \in R_{+}$such that $\mathbf{U}_{\ell, t_{0}} \neq \emptyset$. By definition, (1) has a proper solution $u \in \mathbf{U}_{\ell, t_{0}}$ satisfying the condition $\left(20_{\ell}\right)$ with some $t_{\ell} \geq t_{0}$. Due to (1), $\left(20_{\ell}\right)$, and $\left(28_{\ell}\right)$, it is obvious that condition (22) holds. Thus by Lemma 5 there exists $t_{2}>t_{1}$ such that conditions $\left(23_{i}\right)-(24)$ with $t_{*}=t_{2}$ are fulfilled. On the other hand, according to Lemma 7 (and its proof), we see that

$$
\begin{array}{r}
u^{(\ell-1)}(t) \\
\geq \frac{1}{(n-\ell) !} \int_{t_{2}}^{t} \int_{s}^{+\infty} \xi^{n-\ell-1} p(\xi)(u(\sigma(\xi)))^{\mu(\xi)} d \xi d s \\
\text { for } t \geq t_{2},
\end{array}
$$

and there exists $t_{*}>t_{2}$ such that relation (30) is fulfilled. Without loss of generality we can assume that $\sigma(t) \geq t_{2}$ for $t \geq t_{*}$. Therefore, by (24), from (58) we have

$$
\begin{aligned}
& u^{(\ell-1)}(t) \\
& \geq \frac{1}{(n-\ell) !} \int_{\sigma_{(-k)}\left(t_{*}\right)}^{t} \int_{s}^{+\infty} \xi^{n-\ell-1}(\sigma(\xi))^{(\ell-1) \mu(\xi)} p(\xi) \\
& \times\left(\frac{1}{\ell !} u^{(\ell-1)}(\sigma(\xi))\right)^{\mu(\xi)} d \xi d s .
\end{aligned}
$$

Assume that $\alpha=1$. Then by (44) and (59), we have

$$
\begin{aligned}
& u^{(\ell-1)}(t) \\
& \geq \frac{1}{(n-\ell) !} \int_{\sigma_{(-k)}\left(t_{*}\right)}^{t} \int_{s}^{+\infty} \xi^{n-\ell-1}(\sigma(\xi))^{(\ell-1) \mu(\xi)} p(\xi) \\
& \quad \times\left(\frac{1}{\ell !} \rho_{k-1, \ell, t_{*}}^{(1)}(\sigma(\xi))\right)^{\mu(\xi)} d \xi d s \text { for } t \geq \sigma_{(-k)}\left(t_{*}\right) .
\end{aligned}
$$

On the other hand, according to $\left(23_{\ell-1}\right)$ and $\left(55_{\ell}\right)$ it is obvious that

$$
\frac{u^{(\ell-1)}(t)}{t} \downarrow 0 \quad \text { for } t \uparrow+\infty .
$$

Therefore, from (60) we get

$$
\begin{aligned}
\lim _{t \rightarrow+\infty} \frac{1}{t} \int_{\sigma_{(-k)}\left(t_{*}\right)}^{t} \int_{s}^{+\infty} \xi^{n-\ell-1}(\sigma(\xi))^{(\ell-1) \mu(\xi)} p(\xi) \\
\times\left(\frac{1}{\ell !} \rho_{k, \ell, t_{*}}^{(1)}(\sigma(\xi))\right)^{\mu(\xi)} d \xi d s=0 .
\end{aligned}
$$


Now assume that $\alpha>1$ and $\delta \in(1, \alpha]$. Then according to (47), $\left(23_{\ell-1}\right)$, and (61), from (59) we have

$$
\begin{aligned}
& u^{(\ell-1)}(t) \\
& \geq \frac{1}{(n-\ell) !} \int_{\sigma_{(-k)}\left(t_{*}\right)}^{t} \int_{s}^{+\infty} \xi^{n-\ell-1}(\sigma(\xi))^{(\ell-1) \mu(\xi)} p(\xi) \\
& \times\left(\frac{1}{\ell !} u^{(\ell-1)}(\sigma(\xi))\right)^{\mu(\xi)-\delta} \\
& \times\left(\frac{1}{\ell !} u^{(\ell-1)}(\sigma(\xi))\right)^{\delta} d \xi d s \\
& \geq \frac{1}{(n-\ell) !} \int_{\sigma_{(-k)}\left(t_{*}\right)}^{t} \int_{s}^{+\infty} \xi^{n-\ell-\delta}(\sigma(\xi))^{\delta+(\ell-1) \mu(\xi)} p(\xi) \\
& \times\left(\frac{1}{\ell !} \rho_{k, \ell, t_{*}}^{(\alpha)}(\sigma(\xi))\right)^{\mu(\xi)-\delta} \\
& \times\left(\frac{1}{\ell !} u^{(\ell-1)}(\xi)\right)^{\delta} d \xi d s \\
& \geq \frac{1}{(n-\ell) !} \int_{\sigma_{(-k)}\left(t_{*}\right)}^{t}\left(\frac{u^{\ell-1}(s)}{\ell !}\right)^{\delta} \\
& \times \int_{s}^{+\infty} \xi^{n-\ell-1-\delta}(\sigma(\xi))^{\delta+(\ell-1) \mu(\xi)} p(\xi) \\
& \times\left(\frac{1}{\ell !} \rho_{k, \ell, t_{*}}^{(\alpha)}(\sigma(\xi))\right)^{\mu(\xi)-\delta} d \xi d s .
\end{aligned}
$$

Thus, we obtain

$$
\begin{aligned}
(v(t))^{\delta} \geq & \frac{1}{(\ell !(n-\ell) !)^{\delta}} \\
& \times\left(\int_{\sigma_{(-k)}\left(t_{*}\right)}^{t} v^{\delta}(s) \int_{s}^{+\infty} \xi^{n-\ell-1-\delta}(\sigma(\xi))^{\delta+(\ell-1) \mu(\xi)}\right. \\
& \left.\times p(\xi)\left(\frac{1}{\ell !} \rho_{k, \ell, t_{*}}^{(\alpha)}(\sigma(\xi))\right)^{\mu(\xi)-\delta} d \xi d s\right)^{\delta},
\end{aligned}
$$

where $v(t)=(1 / \ell !) u^{(\ell-1)}(t)$.

It is obvious that there exist $t_{1}>\sigma_{(-k)}\left(t_{*}\right)$ such that

$$
\begin{array}{r}
\int_{\sigma_{(-k)}\left(t_{*}\right)}^{t} v^{\delta}(s) \int_{s}^{+\infty} \xi^{n-\ell-1-\delta}(\sigma(\xi))^{\delta+(\ell-1) \mu(\xi)} p(\xi) \\
\times\left(\frac{1}{\ell !} \rho_{k, \ell, t_{*}}^{(\alpha)}(\sigma(\xi))\right)^{\mu(\xi)-\delta} d \xi d s>0 \\
\text { for } t \geq t_{1} .
\end{array}
$$

Therefore, from (64)

$$
\begin{aligned}
& \int_{t_{1}}^{t} \frac{\varphi^{\prime}(s)}{(\varphi(s))^{\delta}} d s \\
& \quad \geq \frac{1}{(\ell !(n-\ell) !)^{\delta}} \\
& \quad \times \int_{t_{1}}^{t} \int_{s}^{+\infty} \xi^{n-\ell-1-\delta}(\sigma(\xi))^{\delta+(\ell-1) \mu(\xi)} \\
& \quad \times p(\xi)\left(\frac{1}{\ell !} \rho_{k, \ell, t_{*}}^{(\alpha)}(\sigma(\xi))\right)^{\mu(\xi)-\delta} d \xi d s,
\end{aligned}
$$

where

$\varphi(t)$

$$
\begin{aligned}
=\int_{\sigma_{(-k)}\left(t_{*}\right)}^{t}(v(s))^{\delta} \int_{s}^{+\infty} & \xi^{n-\ell-1-\delta}(\sigma(\xi))^{\delta+(\ell-1) \mu(\xi)} \\
& \times p(\xi)\left(\frac{1}{\ell !} \rho_{k, \ell, t_{*}}^{(\alpha)}(\sigma(\xi))\right)^{\mu(\xi)-\delta} d \xi d s .
\end{aligned}
$$

From the last inequality we get

$$
\begin{aligned}
& \int_{t_{1}}^{t} \int_{s}^{+\infty} \xi^{n-\ell-1-\delta}(\sigma(\xi))^{\delta+(\ell-1) \mu(\xi)} p(\xi) \\
& \quad \times\left(\frac{1}{\ell !} \rho_{k, \ell, t_{*}}^{(\alpha)}(\sigma(\xi))\right)^{\mu(\xi)-\delta} d \xi d s \\
& \leq \frac{(\ell !(n-\ell) !)^{\delta}}{\delta-1}\left[\varphi^{1-\delta}\left(t_{1}\right)-\varphi^{1-\delta}(t)\right] \\
& \leq \frac{(\ell !(n-\ell) !)^{\delta}}{\delta-1} \varphi^{1-\delta}\left(t_{1}\right) \quad \text { for } t \geq t_{1} .
\end{aligned}
$$

Passing to the limit in the latter inequality, we get

$$
\begin{aligned}
& \int_{t_{1}}^{+\infty} \int_{s}^{+\infty} \xi^{n-\ell-1-\delta}(\sigma(\xi))^{\delta+(\ell-1) \mu(\xi)} p(\xi) \\
& \quad \times\left(\frac{1}{\ell !} \rho_{k, \ell, t_{*}}^{(\alpha)}(\sigma(\xi))\right)^{\mu(\xi)-\delta} d \xi d s<+\infty ;
\end{aligned}
$$

that is, according to (62) and (69), (56) and (57) hold, which proves the validity of the theorem.

Using Lemma 8 in a similar manner one can prove the following.

Theorem 11. Let $\ell \in\{1, \ldots, n-1\}$ with $\ell+n$ be odd, let (2), (3), $\left(28_{\ell}\right)$, and $\left(55_{\ell}\right)$ be fulfilled, and for some $t_{0} \in R_{+}, \mathbf{U}_{\ell, t_{0}}=\emptyset$. Then there exists $t_{*}>t_{0}$ such that if $\beta=1$, for any $k \in N$

$$
\begin{aligned}
& \limsup _{t \rightarrow+\infty} \frac{1}{t} \\
& \times \int_{\sigma_{(-k)}\left(t_{*}\right)}^{t} \int_{s}^{+\infty} \xi^{n-\ell-1}(\sigma(\xi))^{(\ell-1) \mu(\xi)}
\end{aligned}
$$

$$
\times\left(\tilde{\rho}_{k, \ell, t_{*}}^{(1)}(\sigma(\xi))\right)^{\mu(\xi)} p(\xi) d \xi d s=0
$$


and if $1<\beta<+\infty$, then for any $k \in N$ and $\delta \in(1, \beta]$

$$
\begin{aligned}
\int_{\sigma_{(-k)}\left(t_{*}\right)}^{+\infty} \int_{s}^{+\infty} \xi^{n-\ell-1-\delta}(\sigma(\xi))^{\ell \mu(\xi)+\delta-\beta} \\
\quad \times\left(\widetilde{\rho}_{k, \ell, t_{*}}^{(\beta)}(\sigma(\xi))\right)^{\beta-\delta} p(\xi) d \xi d s<+\infty,
\end{aligned}
$$

where $\beta$ is defined by the second equality of (26) and $\tilde{\rho}_{k, \ell, t_{*}}^{(\beta)}$ is given by (51)-(53).

\section{Sufficient Conditions for Nonexistence of Solutions of the Type $\left(20_{\ell}\right)$}

Theorem 12. Let $\beta<+\infty, \ell \in\{1, \ldots, n-1\}$ with $\ell+n$ odd, let the conditions (2), (3), $\left(28_{\ell}\right)$, and $\left(55_{\ell}\right)$ be fulfilled, and if $\alpha=1$, for any large $t_{*} \in R_{+}$and for some $k \in N$

$$
\begin{aligned}
\limsup _{t \rightarrow+\infty} \frac{1}{t} \int_{\sigma_{(-k)}\left(t_{*}\right)}^{t} \int_{s}^{+\infty} & \xi^{n-\ell-1}(\sigma(\xi))^{(\ell-1) \mu(\xi)} \\
& \times\left(\frac{1}{\ell !} \rho_{k, \ell, t_{*}}^{(1)}(\sigma(\xi))\right)^{\mu(\xi)} p(\xi) d \xi d s>0
\end{aligned}
$$

or if $\alpha>1$, for same $k \in N$ and $\delta \in(1, \alpha]$

$$
\begin{aligned}
\int_{\sigma_{(-k)}\left(t_{*}\right)}^{+\infty} \int_{s}^{+\infty} \xi^{n-\ell-1-\delta}(\sigma(\xi))^{\delta+(\ell-1) \mu(\xi)} \\
\quad \times\left(\frac{1}{\ell !} \rho_{k, \ell, t_{*}}^{(\alpha)}(\sigma(\xi))\right)^{\mu(\xi)-\delta} p(\xi) d \xi d s=+\infty .
\end{aligned}
$$

Then for any $t_{0} \in R_{+}$one has $\mathbf{U}_{\ell, t_{0}}=\emptyset$, where $\alpha$ and $\beta$ are defined by (26) and $\rho_{k, \ell, t_{*}}^{(\alpha)}$ is given by (30)-(32).

Proof. Assume the contrary. Let there exist $t_{0} \in R_{+}$such that $\mathbf{U}_{\ell, t_{0}} \neq \emptyset$ (see Definition 6). Then (1) has a proper solution $u:\left[t_{0},+\infty\right) \rightarrow R$ satisfying the condition $\left(20_{\ell}\right)$. Since the condition of Theorem 10 is fulfilled, there exists $t_{*}>t_{0}$ such that if $\alpha=1$ (if $\alpha>1$ ), the condition (56) (the condition (57)) holds, which contradicts $\left(72_{\ell}\right)$ and $\left(73_{\ell}\right)$. The obtained contradiction proves the validity of the theorem. ing.

Using Theorem 11 analogously we can prove the follow-

Theorem 13. Let $\ell \in\{1, \ldots, n-1\}$ with $\ell+n$ odd, let the conditions (2), (3), $\left(28_{\ell}\right)$, and $\left(55_{\ell}\right)$ be fulfilled, and if $\beta=1$, for any large $t_{*} \in R_{+}$and for some $k \in N$

$$
\begin{aligned}
\limsup _{t \rightarrow+\infty} \frac{1}{t} \int_{\sigma_{(-k)}\left(t_{*}\right)}^{t} \int_{s}^{+\infty} \xi^{n-\ell-1}(\sigma(\xi))^{(\ell-1) \mu(\xi)} \\
\quad \times\left(\frac{1}{\ell !} \tilde{\rho}_{k, \ell, t_{*}}^{(1)}(\sigma(\xi))\right)^{\mu(\xi)} p(\xi) d \xi d s>0
\end{aligned}
$$

or if $1<\beta<+\infty$ for same $k \in N$ and $\delta \in(1, \beta]$

$$
\begin{aligned}
\int_{\sigma_{(-k)}\left(t_{*}\right)}^{+\infty} \int_{s}^{+\infty} \xi^{n-\ell-1-\delta}(\sigma(\xi))^{\ell \mu(\xi)+\delta-\beta} \\
\quad \times\left(\widetilde{\rho}_{k, \ell, t_{*}}^{(\beta)}(\sigma(\xi))\right)^{\beta-\delta} p(\xi) d \xi d s=+\infty .
\end{aligned}
$$

Then for any $t_{0} \in R_{+}$we have $\mathbf{U}_{\ell, t_{0}}=\emptyset$, where $\beta$ is defined by the second equality of (26) and $\tilde{\rho}_{k, \ell, t_{*}}$ is given by (51)-(53).

Corollary 14. Let $\ell \in\{1, \ldots, n-1\}$ with $\ell+n$ odd, let the conditions (2), (3), and $\left(55_{\ell}\right)$ be fulfilled, $\alpha=1, \beta<+\infty$, and

$$
\limsup _{t \rightarrow+\infty} \frac{1}{t} \int_{0}^{t} \int_{s}^{+\infty} \xi^{n-\ell-1}(\sigma(\xi))^{(\ell-1) \mu(\xi)} p(\xi) d \xi d s>0 . \quad\left(76_{\ell}\right)
$$

Then $\mathbf{U}_{\ell, t_{0}}=\emptyset$ for any $t_{0} \in R_{+}$.

Proof. Since

$$
\rho_{1, \ell, t}^{(\alpha)}\left(\sigma\left(t_{*}\right)\right) \geq \ell ! \quad \text { for } t \geq \sigma_{(-1)}\left(t_{*}\right)
$$

it suffices to note that by $\left(76_{\ell}\right)$ the conditions $\left(72_{\ell}\right)$ and $\left(28_{\ell}\right)$ are fulfilled for $k=1$.

Corollary 15. Let $\ell \in\{1, \ldots, n-1\}$ with $\ell+n$ odd, let the conditions (2) and (3) be fulfilled, $\alpha=1, \beta<+\infty$, and

$$
\liminf _{t \rightarrow+\infty} t \int_{t}^{+\infty} s^{n-\ell-2}(\sigma(s))^{1+(\ell-1) \mu(s)} p(s) d s=\gamma>0 .
$$

If, moreover, for some $\varepsilon \in(0, \gamma)$

$$
\begin{aligned}
& \limsup _{t \rightarrow+\infty} \frac{1}{t} \\
& \quad \times \int_{0}^{t} \int_{s}^{+\infty} \xi^{n-\ell-1}(\sigma(\xi))^{\mu(\xi)(\ell-1+((\gamma-\varepsilon) /(\ell !(n-\ell) !)))} p(\xi) d \xi d s \\
& \quad>0,
\end{aligned}
$$

then for any $t_{0} \in R_{+}, \mathbf{U}_{\ell, t_{0}}=\emptyset$.

Proof. Clearly by virtue of $\left(78_{\ell}\right)$ conditions $\left(28_{\ell}\right)$ and $\left(55_{\ell}\right)$ are fulfilled. Let $\varepsilon \in(0, \gamma)$. According to $\left(78_{\ell}\right)$ and $\left(79_{\ell}\right)$ it is obvious that, for large $t, \rho_{1, \ell, t_{*}}^{(1)}(t) \geq \ell ! t^{(\gamma-\varepsilon) /(\ell !(n-\ell) !)}$. Therefore, by $\left(79_{\ell}\right)$, for $k=1,\left(72_{\ell}\right)$ holds, which proves the validity of the corollary.

Corollary 16. Let $\ell \in\{1, \ldots, n-1\}$ with $\ell+n$ odd, let the conditions (2), (3), $\left(28_{\ell}\right)$, and $\left(55_{\ell}\right)$ be fulfilled, $\alpha>1, \beta<$ $+\infty$, and for some $\delta \in(1, \alpha]$

$$
\int_{0}^{+\infty} \int_{s}^{+\infty} \xi^{n-\ell-1-\delta}(\sigma(\xi))^{\delta+(\ell-1) \mu(\xi)} p(\xi) d \xi d s=+\infty
$$

Then for any $t_{0} \in R_{+}, \mathbf{U}_{\ell, t_{0}}=\emptyset$, where $\alpha$ is defined by the first condition of (3) 
Proof. By $\left(80_{\ell}\right)$ and $(77)$, for $k=1$, the condition $\left(73_{\ell}\right)$ holds, which proves the validity of the corollary.

Corollary 17. Let $\ell \in\{1, \ldots, n-1\}$ with $\ell+n$ odd and let the conditions (2), (3), and $\left(78_{\ell}\right)$ be fulfilled. Moreover, if $\alpha>1$, $\beta<+\infty$, and there exists $m \in N$ such that

$$
\liminf _{t \rightarrow+\infty} \frac{\sigma^{m}(t)}{t}>0
$$

then for any $t_{0} \in R_{+}, \mathbf{U}_{\ell, t_{0}}=\emptyset$, where $\alpha$ is defined by the first condition of (3).

Proof. By $\left(78_{\ell}\right)$ there exist $c>0$ and $t_{1} \in R_{+}$such that

$$
t \int_{t}^{+\infty} \xi^{n-\ell-2}(\sigma(\xi))^{1+(\ell-1) \mu(\xi)} p(\xi) d \xi \geq c \quad \text { for } t \geq t_{1}
$$

Let $\delta=(1+\alpha) / 2$ and $M=m(1+\alpha) / c(\alpha-\delta)$. Then by $\left(82_{\ell}\right)$ and (30), for large $t_{*}>t_{1}$,

$$
\rho_{1, \ell, t_{*}}^{(\alpha)}(t) \geq t^{M c} \quad t \geq t_{*} .
$$

Therefore

$$
\begin{gathered}
\left(\frac{\sigma(t)}{t}\right)^{\delta}\left(\frac{1}{\ell !} \rho_{1, \ell, t_{*}}^{(\alpha)}(\sigma(t))\right)^{\mu(t)-\delta} \\
\geq\left(\frac{\sigma(t)}{t}\right)^{\delta}\left(\frac{1}{\ell !} \sigma^{M c}(t)\right)^{\alpha-\delta} \\
=\frac{1}{(\ell !)^{\alpha-\delta}}\left(\frac{(\sigma(t))^{1+(M c(\alpha-\delta) / \delta)}}{t}\right)^{\delta} \\
>\frac{1}{(\ell !)^{\alpha-\delta}}\left(\frac{(\sigma(t))^{m}}{t}\right)^{\delta} .
\end{gathered}
$$

Thus, by $(81)$ and $\left(78_{\ell}\right)$, it is obvious that $\left(73_{\ell}\right)$ holds, which proves the corollary.

Quite similarly one can prove the following.

Corollary 18. Let $\ell \in\{1, \ldots, n-1\}$ with $\ell+n$ odd, let the conditions (2), (3), $\left(28_{\ell}\right)$, and $\left(55_{\ell}\right)$ be fulfilled, $\alpha>1$, and $\beta<+\infty$. Moreover, if

$$
\liminf _{t \rightarrow+\infty} t \ln t \int_{t}^{+\infty} \xi^{n-\ell-2}(\sigma(\xi))^{1+(\ell-1) \mu(\xi)} p(\xi) d \xi>0
$$

and for some $\delta \in(1, \alpha]$ and $m \in N$

$$
\int_{0}^{+\infty} \int_{s}^{+\infty} \xi^{n-\ell-1-\delta}(\sigma(\xi))^{\delta+(\ell-1) \mu(\xi)}(\ln \sigma(\xi))^{m} d \xi d s=+\infty
$$

then for any $t_{0} \in R_{+}$one has $\mathbf{U}_{\ell, t_{0}}=\emptyset$, where $\alpha$ and $\beta$ are defined by the condition of (26).
Corollary 19. Let $\ell \in\{1, \ldots, n-1\}$ with $\ell+n$ be odd, let the conditions (2), (3), and $\left(28_{\ell}\right)$ be fulfilled, $\alpha>1$, and $\beta<+\infty$. Moreover, let there exist $\gamma \in(0,1)$ and $r \in(0,1]$ such that

$$
\begin{gathered}
\liminf _{t \rightarrow+\infty} t^{\gamma} \int_{t}^{+\infty} \xi^{n-\ell-1}(\sigma(\xi))^{(\ell-1) \mu(\xi)} p(\xi) d \xi>0, \\
\liminf _{t \rightarrow+\infty} \frac{\sigma(t)}{t^{r}}>0
\end{gathered}
$$

and let at least one of the conditions

$$
r \alpha \geq 1
$$

or $r \alpha<1$ and for some $\varepsilon>0$ and $\delta \in(1, \alpha]$

$$
\begin{aligned}
& \int_{0}^{+\infty} \int_{s}^{+\infty} \xi^{n-\ell-1-\delta+(\alpha r(1-\gamma) /(1-\alpha r))-\varepsilon} \\
& \quad \times(\sigma(\xi))^{(\ell-1) \mu(\xi)} p(\xi) d \xi d s=+\infty
\end{aligned}
$$

be fulfilled. Then for any $t_{0} \in R_{+}$one has $\mathbf{U}_{\ell, t_{0}}=\emptyset$, where $\alpha$ is defined by (26).

Proof. It suffices to show that the condition $\left(73_{\ell}\right)$ is satisfied for some $k \in N$ and $\delta=(1+\alpha) / 2$. Indeed, according to $\left(87_{\ell}\right)$ and (88), there exist $\gamma \in(0,1), r \in(0,1], c>0$, and $t_{1} \in R_{+}$ such that

$$
\begin{gathered}
t^{\gamma} \int_{t}^{+\infty} \xi^{n-\ell-1}(\sigma(\xi))^{(\ell-1) \mu(\xi)} p(\xi) d \xi>c \quad \text { for } t \geq t_{1}, \\
\sigma(t) \geq c t^{r} \quad \text { for } t \geq t_{1} .
\end{gathered}
$$

By (77), (31), and (91), from (31) we get

$$
\begin{aligned}
& \rho_{2, \ell, t_{*}}^{(\alpha)}(t) \\
& \quad \geq \frac{c}{(n-\ell) !} \int_{\sigma_{(-1)}\left(t_{*}\right)}^{t} s^{-\gamma} d s \\
& \quad=\frac{c\left(t^{1-\gamma}-\sigma_{(-1)}^{1-\gamma}\left(t_{*}\right)\right)}{(n-\ell) !(1-\gamma)} \text { for } t \geq \sigma_{(-1)}\left(t_{*}\right) .
\end{aligned}
$$

Let $\gamma_{1} \in(\gamma, 1)$. Choose $t_{2}>\sigma_{(-1)}\left(t_{*}\right)$ such that

$$
\rho_{2, \ell, t_{*}}^{(\alpha)}(t) \geq t^{1-\gamma_{1}} \quad \text { for } t \geq t_{2}
$$

Therefore, by (91) from (31) we can find $t_{3}>t_{2}$ such that

$$
\rho_{3, \ell, t_{*}}^{(\alpha)}(t) \geq t^{\left(1-\gamma_{1}\right)(1+\alpha r)} \quad \text { for } t \geq t_{3} .
$$

Hence for any $k_{0} \in N$ there exists $t_{k_{0}}$ such that

$$
\rho_{k_{0}, \ell, t_{*}}^{(\alpha)}(t) \geq t^{\left(1-\gamma_{1}\right)\left(1+\alpha r+\cdots+(\alpha r)^{k_{\alpha}-2}\right)} \quad \text { for } t \geq t_{k_{0}} .
$$

Assume that (89) is fulfilled. Choose $k_{0} \in N$ such that $k_{0}-1 \geq((1-r)(1+\alpha)) /\left(\left(1-\gamma_{1}\right)(\alpha-1)\right)$. Then according 
to (92), (96), and $\left(28_{\ell}\right)$, the condition $\left(73_{\ell}\right)$ holds for $k=k_{0}$ and $\delta=(1+\alpha) / 2$. In this case, the validity of the corollary has already been proven.

Assume now that $\left(90_{\ell}\right)$ is fulfilled. Let $\varepsilon>0$ and choose $k_{0} \in N$ and $\gamma_{1} \in(\gamma, 1)$ such that

$$
\left(1-\gamma_{1}\right)\left(1+\alpha r+\cdots+(\alpha r)^{k_{0}-2}\right)>\frac{(1-\gamma) \alpha r}{1-\alpha r}-\varepsilon .
$$

Then according to (96), (92), and $\left(90_{\ell}\right)$, it is obvious that $\left(73_{\ell}\right)$ holds for $k=k_{0}$. The proof of the corollary is complete.

Using Theorem 13, in a manner similar to above we can prove the following.

Corollary 20. Let $\ell \in\{1, \ldots, n-1\}$ with $\ell+n$ odd, let the conditions (2), (3), and $\left(28_{\ell}\right)$ be fulfilled, $\beta=1$, and

$$
\limsup _{t \rightarrow+\infty} \frac{1}{t} \int_{0}^{t} \int_{s}^{+\infty} \xi^{n-\ell-1}(\sigma(\xi))^{(\ell-1) \mu(\xi)} p(\xi) d \xi d s>0 .
$$

Then for any $t_{0} \in R_{+}, \mathbf{U}_{\ell, t_{0}}=\emptyset$, where $\beta$ is given by (26).

Corollary 21. Let $\ell \in\{1, \ldots, n-1\}$ with $\ell+n$ odd, let the conditions (2), (3), and $\left(28_{\ell}\right)$ be fulfilled, $\beta=1$, and

$$
\liminf _{t \rightarrow+\infty} t \int_{t}^{+\infty} \xi^{n-\ell-1}(\sigma(\xi))^{(\ell-1) \mu(\xi)} p(\xi) d \xi=\gamma>0 .
$$

Moreover, let for some $\varepsilon \in(0, \gamma)$

$$
\begin{aligned}
\limsup _{t \rightarrow+\infty} \frac{1}{t} \int_{0}^{t} \int_{s}^{+\infty} & \xi^{n-\ell-1}(\sigma(\xi))^{\mu(\xi)(\ell-1+((\gamma-\varepsilon) / \ell !(n-\ell) !))} \\
& \times p(\xi) d \xi d s>0 .
\end{aligned}
$$

Then for any $t_{0} \in R_{+}$one has $\mathbf{U}_{\ell, t_{0}}=\emptyset$, where $\beta$ is given by (26).

Corollary 22. Let $\ell \in\{1, \ldots, n-1\}$ with $\ell+n$ odd, let the conditions (2), (3), $\left(28_{\ell}\right)$, and $\left(55_{\ell}\right)$ be fulfilled, $1<\beta<+\infty$, and for some $\delta \in(1, \beta]$

$$
\int_{0}^{+\infty} \int_{s}^{+\infty} \xi^{n-\ell-1-\delta}(\sigma(\xi))^{\ell \mu(\xi)+\delta-\beta} p(\xi) d \xi d s=+\infty .
$$

Then for any $t_{0} \in R_{+}, \mathbf{U}_{\ell, t_{0}}=\emptyset$.

\section{Differential Equation with Property A}

Theorem 23. Let the conditions (2), (3) be fulfilled and for any $\ell \in\{1, \ldots, n-1\}$ with $\ell+n$ odd, the conditions $\left(74_{\ell}\right)$ and $\left(75_{\ell}\right)$ hold. Moreover for any large $t_{*} \in R_{+}$, if $\alpha=1$ and $\beta<+\infty$ for some $k \in N$ let $\left(72_{\ell}\right)$ be fulfilled or if $\alpha>1$ and $\beta<+\infty$, for some $k \in N, M \in(1,+\infty)$, and $\delta \in(1, \alpha]$, let $\left(72_{\ell}\right)$ be fulfilled. Then, if for odd $n$

$$
\int_{0}^{+\infty} t^{n-1} p(t) d t=+\infty
$$

then (1) has Property A, where $\alpha$ and $\beta$ are defined by (26) and $\rho_{k, \ell, t_{*}}^{(\alpha)}$ is given by (30)-(32).
Proof. Let (1) have a proper nonoscillatory solution $u$ : $\left[t_{0},+\infty\right) \rightarrow(0,+\infty)$ (the case $u(t)<0$ is similar). Then by (2), (3), and Lemma 3 there exists $\ell \in\{0, \ldots, n-1\}$ such that $\ell+n$ is odd and conditions $\left(20_{\ell}\right)$ hold. Since, for any $\ell \in$ $\{1, \ldots, n-1\}$ with $\ell+n$ odd, the conditions of Theorem 12 are fulfilled we have $\ell \notin\{1, \ldots, n-1\}$. Now assume that $\ell=0$, $n$ is odd, and there exists $c \in(0,1)$ such that $u(t) \geq c$ for sufficiently large $t$. According to $\left(20_{0}\right)$ since $\beta<+\infty$, from (1) we have

$$
\begin{aligned}
& \sum_{i=0}^{n-1}(n-i-1) ! t_{1}^{i}\left|u^{(i)}\left(t_{1}\right)\right| \\
& \quad \geq \int_{t_{1}}^{t} s^{n-1} p(s) c^{\mu(s)} d s \\
& \geq c^{\beta} \int_{t_{1}}^{t} s^{n-1} p(s) d s \quad \text { for } t \geq t_{1},
\end{aligned}
$$

where $t_{1}$ is a sufficiently large number. The last inequality contradicts the condition (102). The obtained contradiction proves that (1) has Property A.

Theorem 24. Let the conditions (2), (3) be fulfilled and for any $\ell \in\{1, \ldots, n-1\}$ with $\ell+n$ odd the conditions $\left(28_{\ell}\right)$ and $\left(55_{\ell}\right)$ hold. Let moreover, if $\beta=1$, for some $k \in N\left(74_{\ell}\right)$ hold or if $1<\beta<+\infty$ and for some $k \in N, M \in(1,+\infty)$, and $\delta \in(1, \beta],\left(75_{\ell}\right)$ hold. Then, if for odd $n$ (102) is fulfilled, then (1) has Property A, where $\beta$ is defined by the second equality of (26) and $\tilde{\rho}_{k, \ell, t_{*}}^{(\beta)}$ is given by (51)-(53).

Proof. The proof of the theorem is analogous to that of Theorem 23. We simply use Theorem 13 instead of Theorem 12.

Theorem 25. Let $\alpha>1, \beta<+\infty$, let the conditions (2), (3), $\left(28_{1}\right)$, and $\left(55_{1}\right)$ be fulfilled, and

$$
\liminf _{t \rightarrow+\infty} \frac{(\sigma(t))^{\mu(t)}}{t}>0
$$

If, moreover, for some $k \in N, M \in(1,+\infty)$, and $\delta \in(1, \alpha]$, $\left(73_{1}\right)$ holds and for odd $n$ (102) is fulfilled, then (1) has Property A, where $\alpha$ and $\beta$ are defined by (26) and $\rho_{k, 1, t_{*}}^{(\alpha)}$ is given by (30)-(32).

Proof. It suffices to note that by $(104)$ and $\left(72_{1}\right)$, for any $\ell \in$ $\{2, \ldots, n-1\}$ there exist $M>1, k \in N$, and $\delta \in(1, \alpha)$ such that condition $\left(72_{\ell}\right)$ is fulfilled.

Theorem 26. Let $1<\beta<+\infty$, and conditions (2), (3), $\left(28_{1}\right)$, (29), and (104) be fulfilled. If, moreover, for some $k \in N$, $M \in(1,+\infty)$, and $\delta \in(1, \beta],\left(75_{1}\right)$ holds and for odd $n(102)$ is fulfilled, then (1) has Property $\mathbf{A}$, where $\beta$ is defined by the second condition of $(26)$ and $\tilde{\rho}_{k, 1, t_{*}}^{(\beta)}$ is given by (51)-(55).

Proof. The theorem is proved similarly to Theorem 25 if we replace the condition $\left(73_{1}\right)$ by the condition $\left(75_{1}\right)$. 
Corollary 27. Let $\alpha=1, \beta<+\infty$, and conditions (2), (3), $\left(28_{1}\right),\left(76_{1}\right)$, and (104) be fulfilled. Then (1) has Property A, where $\alpha$ and $\beta$ are given by (26).

Proof. By (28 $),\left(76_{1}\right)$, and (104), condition (102), and for any $\ell \in\{1, \ldots, n-1\}\left(76_{\ell}\right)$ holds. Now assume that (1) has a proper nonoscillatory solution $u:\left[t_{0},+\infty\right) \rightarrow(0,+\infty)$. Then, by (2), (3), and Lemma 3, there exists $\ell \in\{0, \ldots, n-1\}$ such that $\ell+n$ is odd and the condition $\left(20_{\ell}\right)$ holds. Since for any $\ell \in\{1, \ldots, n-1\}$ with $\ell+n$ odd the conditions of Corollary 14 are fulfilled, we have $\ell \notin\{1, \ldots, n-1\}$. Therefore $n$ is odd and $\ell=0$. According to (102) and $\left(20_{0}\right)$ it is obvious that the condition (5) holds. Therefore, (1) has Property A.

Using Corollaries 15-19, the validity of Corollaries 28-32 can be proven similarly to Corollary 27.

Corollary 28. Let $\alpha=1, \beta<+\infty$, and conditions (2), (3), $\left(78_{1}\right),\left(79_{1}\right)$, and (104) be fulfilled. Then (1) has Property A, where $\alpha$ and $\beta$ are given by (26).

Corollary 29. Let $\alpha>1, \beta<+\infty$, conditions (2), (3), (104) be fulfilled, and $\left(80_{1}\right)$ for some $\delta \in(1, \alpha]$ hold and if $n$ is odd, condition (102) holds. Then (1) has Property A.

Corollary 30. Let $\alpha>1, \beta<+\infty$, the conditions (2), (3), $\left(28_{1}\right),\left(78_{1}\right),(104)$ be fulfilled, and (81) for some $m \in N$ holds. Then (1) has Property A.

Corollary 31. Let $\alpha>1, \beta<+\infty$, the conditions (2), (3), $\left(28_{1}\right),(104)$, and $\left(85_{1}\right)$ and for some $\delta \in(1, \alpha]$ and $m \in$ $N\left(86_{1}\right)$ be fulfilled. Then (1) has Property $\mathbf{A}$, where $\alpha$ and $\beta$ are given by (26).

Corollary 32. Let $\alpha>1, \beta<+\infty$, and the conditions (2), (3), (281), and (104) be fulfilled. Let moreover, there exist $\gamma \in$ $(0,1)$ and $r \in(0,1]$ such that $\left(87_{1}\right)$ and $(88)$ hold. Then either condition (89) or condition $\left(90_{1}\right)$ is sufficient for (1) to have Property A.

Corollary 33. Let $\beta=1$ and the conditions (2), (3), $\left(28_{1}\right)$, (104), and $\left(98_{1}\right)$ be fulfilled. Then (1) has Property A, where $\beta$ is defined by the second condition of (26).

Proof. By $\left(28_{1}\right),(104)$, and $\left(98_{1}\right)$, the condition (102), and for any $\ell \in\{1, \ldots, n-1\}\left(98_{\ell}\right)$ holds. Therefore, by Corollary 20 , for any $t_{0} \in R_{+}$and $\ell \in\{1, \ldots, n-1\}$ with $\ell+n$ is odd $\mathbf{U}_{\ell, t_{0}}=$ $\emptyset$. On the other hand, if $n$ is odd and $\ell=0$, according to (102) it is obvious that the condition (5) holds, which proves that (1) has Property A.

Using Corollaries 21 and 22, we can analogously prove the following corollaries.

Corollary 34. Let $\beta=1$ and the conditions (2), (3), (104), $\left(99_{1}\right)$, and $\left(100_{1}\right)$ be fulfilled. Then (1) has Property A, where $\beta$ is given by (26).

Corollary 35. Let $1<\beta<+\infty$, the conditions (2), (3), (104), (28 $)$, and (29, be fulfilled, and if $n$ is odd (102) holds.
Moreover, if for some $\delta \in(1, \beta)\left(101_{1}\right)$ holds, then (1) has Property A.

Theorem 36. Let $\alpha>1, \beta<+\infty$, the conditions (2), (3), $\left(28_{n-1}\right)$, and $\left(29_{n-1}\right)$ be fulfilled, and

$$
\limsup _{t \rightarrow+\infty} \frac{(\sigma(t))^{\mu(t)}}{t}<+\infty .
$$

Moreover, for some $k \in N, M \in(1,+\infty)$, and $\delta \in(1, \alpha]$, let $\left(73_{n-1}\right)$ be fulfilled. Then (1) has Property $\mathbf{A}$, where $\alpha$ and $\beta$ are defined by (26).

Proof. It suffices to note that by $\left(28_{n-1}\right),\left(29_{n-1}\right),(105)$, and $\left(73_{n-1}\right)$ there exist $M>1, k \in N$, and $\delta \in(1, \alpha]$ such that $\left(28_{\ell}\right),\left(29_{\ell}\right)$, and $\left(73_{\ell}\right)$ hold for any $\ell \in\{1, \ldots, n-2\}$.

Theorem 37. Let $1<\beta<+\infty$ and the conditions (2), (3), $\left(28_{n-1}\right),\left(29_{n-1}\right)$, and (105) be fulfilled, and for some $k \in N$, $M \in(1,+\infty)$, and $\delta \in(1, \beta],\left(75_{n-1}\right)$ holds. Then (1) has Property $\boldsymbol{A}$, where $\beta$ is given by the second condition of (26).

Proof. The proof is similar to that of Theorem 36 we replace the condition $\left(73_{n-1}\right)$ by the condition $\left(75_{n-1}\right)$.

Corollary 38. Let $\alpha=1, \beta<+\infty$, and the conditions (2), (3), $\left(28_{n-1}\right),\left(76_{n-1}\right)$, and (105) be fulfilled. Then (1) has Property A, where $\alpha$ and $\beta$ are given by (26).

Proof. By $\left(28_{n-1}\right),\left(76_{n-1}\right)$, and (105), the condition (102), and for any $\ell \in\{1, \ldots, n-1\}$ the condition $\left(76_{\ell}\right)$ holds; it is obvious that (1) has Property A.

Using Corollaries 15-19, the validity of Corollaries 39-43 below can be proven similarly to Corollary 38 .

Corollary 39. Let $\alpha=1, \beta<+\infty$, and the conditions (2), (3), $\left(78_{n-1}\right),\left(79_{n-1}\right)$, and (105) be fulfilled. Then (1) has Property A, where $\alpha$ and $\beta$ are given by (26).

Corollary 40. Let $\alpha>1, \beta<+\infty$, and the conditions (2), (3), (105), and, for some $\delta \in(1, \alpha],\left(80_{n-1}\right)$ be fulfilled. Then (1) has Property A, where $\alpha$ is given by (26).

Corollary 41. Let $\alpha>1, \beta<+\infty$, and the conditions (2), (3), $\left(28_{n-1}\right),\left(78_{n-1}\right),(105)$, and for some $m \in N(81)$ be fulfilled. Then (1) has Property A, where $\alpha$ and $\beta$ are given by (26).

Corollary 42. Let $\alpha>1, \beta<+\infty$, and the conditions (2), (3), $\left(28_{n-1}\right),\left(85_{n-1}\right)$ and $(105)$ be fulfilled and for some $\delta \in(1, \alpha]$ and $m \in N\left(86_{n-1}\right)$ holds. Then (1) has Property A, where $\alpha$ and $\beta$ are given by (26).

Corollary 43. Let $\alpha>1, \beta<+\infty$, and the conditions (2), (3), $\left(28_{n-1}\right)$, and (105) be fulfilled. Let, moreover, there exist $\gamma \in(0,1)$ and $r \in(0,1)$ such that $\left(87_{n-1}\right)$ and $(88)$ hold. Then either condition (89) or condition $\left(90_{n-1}\right)$ is sufficient for (1) to have Property $\mathbf{A}$.

Corollary 44. Let $\beta=1$ and the conditions (2), (3), $\left(28_{n-1}\right)$, (105), and $\left(98_{n-1}\right)$ be fulfilled. Then (1) has Property A. 
Proof. By $\left(28_{n-1}\right),(105)$, and $\left(98_{n-1}\right)$, the conditions (102), and for any $\ell \in\{1, \ldots, n-1\}\left(98_{\ell}\right)$ holds. Therefore, by Corollary 20, it is clear that (1) has Property A.

Using Corollaries 21 and 22, analogously we can prove Corollaries 45 and 5.18.

Corollary 45. Let $\beta=1$ and the conditions (2), (3), (105), $\left(99_{n-1}\right)$, and $\left(100_{n-1}\right)$ be fulfilled. Then (1) has Property A, where $\beta$ is given by (26).

Corollary 46. Let $1<\beta<+\infty$ and the conditions (2), (3), (105), and $\left(29_{n-1}\right)$ be fulfilled. If for some $\delta \in(1, \beta],\left(101_{n-1}\right)$ holds, then (1) has Property A.

\section{Necessary and Sufficient Conditions}

Theorem 47. Let $\alpha>1$ and $\beta<+\infty$, let the conditions (2) and (3) be fulfilled and

$$
\liminf _{t \rightarrow+\infty} \frac{\sigma(t)}{t}>0
$$

Then the condition (102) is necessary and sufficient for (1) to have Property A, where $\alpha$ and $\beta$ are given by (26).

Proof. Necessity. Assume that (1) has Property A and

$$
\int_{0}^{+\infty} t^{n-1} p(t) d t<+\infty .
$$

Therefore, by Lemma 4.1 from [28], there exists $c \neq 0$ such that (1) has a proper solution $u:[0,+\infty) \rightarrow R$ satisfying the condition $\lim _{t \rightarrow+\infty} u(t)=c$. But this contradicts the fact that (1) has Property A.

Sufficiency. By (106) and (102) it is obvious that the condition $\left(80_{1}\right)$ holds. Therefore the sufficiency follows from Corollary 29.

Remark 48. In Theorem 47 the condition $\beta<+\infty$ cannot be replaced by the condition $\beta=+\infty$. Indeed, let $c \in(0,1 / 2)$, $\alpha=1 / 2 c$, and

$$
p(t)=\frac{n ! t^{\lg _{\alpha} t}}{t^{1+n}(1+c t)^{\lg _{\alpha} t}} \quad t \geq 1 .
$$

It is obvious that the condition (102) is fulfilled, but equation

$$
u^{(n)}(t)+p(t)|u(t)|^{\lg _{\alpha} t} \operatorname{sign} u(t)=0
$$

has solution $u(t)=(1 / t+c)$. Therefore, (109) does not have Property A.

\section{Conflict of Interests}

The authors declare that there is no conflict of interests regarding the publication of this paper.

\section{Acknowledgment}

The work was supported by the Sh. Rustaveli National Science Foundation Grant no. 31/09.

\section{References}

[1] I. J. H. Lane, "On the theoretical temperature of the sun under the hypothesis of a gaseous mass maintaining its volume by its internal heat and depending on the laws of gases known to terrestial experiment," American Journal of Science and Arts, vol. 4, pp. 57-74, 1870.

[2] R. H. Fowler, "Further studies of Emden's and similar differential equations," Quarterly Journal of Mathematics, vol. 2, no. 1, pp. 259-288, 1931.

[3] R. Bellman, Stability Theory of Differential Equations, MaGrawHill, New York, NY, USA, 1953.

[4] G. Sansone, Equazioni Differenziali nel Campo Reale. II, Zanichelli, Bologna, Italy, 3rd edition, 1963.

[5] R. Conti, D. Graffi, and G. Sansone, "The Italian contribution to the theory of non-linear ordinary differential equations and to nonlinear mechanics during the years 1951-1961," in Qualitative Methods in the Theory of Nonlinear Vibrations II, pp. 172-189, 1961.

[6] F. V. Atkinson, “On second order nonlinear oscillation," Pacific Journal of Mathematics, vol. 5, no. 5, pp. 643-647, 1955.

[7] J. Kurzweil, "A note on oscillatory solutions of the equation $y^{\text {" }+}$ $f(x) y^{2 n-1}=0$," Časopis Pro Pěstování Matematiky, vol. 85, pp. 357-358, 1960.

[8] I. T. Kiguradze and T. A. Chanturia, Asymptotic Properties of Solutions of Nonautonomous Ordinary Differential Equations, Nauka, Moscow, Russia, 1990.

[9] S. Belohorec, "Oscillatory solutions of certain nonlinear differential equations of second order," Matematicko-Fyzikálny Časopis, vol. 11, pp. 250-255, 1961.

[10] R. S. Dahiya and B. Singh, "On oscillatory behavior of even order delay equations," Journal of Mathematical Analysis and Applications, vol. 42, no. 1, pp. 183-190, 1973.

[11] R. G. Koplatadze, "On oscillatory solutions of second order delay differential inequalities," Journal of Mathematical Analysis and Applications, vol. 42, no. 1, pp. 148-157, 1973.

[12] R. G. Koplatadze, "On some properties of solutions of nonlinear differential inequalities and equations with a delayed argument," Differentsial'nye Uravneniya, vol. 12, no. 11, pp. 19711984, 1976 (Russian).

[13] R. G. Koplatadze and T. A. Chanturia, On Some Properties of Solutions of Nonlinear Differential Inequalities and Equations with a Delayed Argument, Tbilisi State University Press, Tbilisi, Georgia, 1977, (Russian).

[14] S. R. Grace and B. S. Lalli, "Oscillation theorems for certain second-order perturbed nonlinear differential equations," Journal of Mathematical Analysis and Applications, vol. 77, no. 1, pp. 205-214, 1980.

[15] S. R. Grace and B. S. Lalli, "Oscillation theorems for nth-order delay differential equations," Journal of Mathematical Analysis and Applications, vol. 91, no. 2, pp. 352-366, 1983.

[16] A. G. Kartsatos, "Recent results on oscillation of solutions of forced and perturbed nonlinear differential equations of even order," in Stability of Dynamic Systems: Theory and Applications, vol. 28 of Lecture Notes in Pure and Applied Mathematics, pp. 1772, Dekker, New York, NY, USA, 1977.

[17] T. Kusano and H. Onose, "Oscillations of functional differential equations with retarded argument," Journal of Differential Equations, vol. 15, no. 2, pp. 269-277, 1974.

[18] W. E. Mahfoud, "Oscillation theorems for a second order delay differential equation," Journal of Mathematical Analysis and Applications, vol. 63, no. 2, pp. 339-346, 1978. 
[19] W. E. Mahfoud, "Oscillation and asymptotic behavior of solutions of Nth order nonlinear delay differential equations," Journal of Differential Equations, vol. 24, no. 1, pp. 75-98, 1977.

[20] U. Sztaba, "Note on the Dahiya and Singh paper: "On oscillatory behavior of even-order delay equations'”' Journal of Mathematical Analysis and Applications, vol. 63, no. 2, pp. 313-318, 1978.

[21] J. R. Graef, R. Koplatadze, and G. Kvinikadze, "Nonlinear functional differential equations with Properties A and B," Journal of Mathematical Analysis and Applications, vol. 306, no. 1, pp. 136-160, 2005.

[22] R. Koplatadze, "Quasi-linear functional differential equations with Property A," Journal of Mathematical Analysis and Applications, vol. 330, no. 1, pp. 483-510, 2007.

[23] R. Koplatadze, "On oscillatory properties of solutions of generalized Emden-Fowler type differential equations," Proceedings of A. Razmadze Mathematical Institute, vol. 145, pp. 117-121, 2007.

[24] R. Koplatadze, "On asymptotic behaviors of solutions of "almost linear" and essential nonlinear functional differential equations," Nonlinear Analysis: Theory, Methods and Applications, vol. 71, no. 12, pp. e396-e400, 2009.

[25] R. Koplatadze and E. Litsyn, "Oscillation criteria for higher order, "almost linear" functional differential equation," Functional Differential Equations, vol. 16, no. 3, pp. 387-434, 2009.

[26] R. Koplatadze, "On asymptotic behavior of solutions of $n$ th order Emden-Fowler differential equations with advanced argument," Czechoslovak Mathematical Journal, vol. 60, no. 3, pp. 817-833, 2010.

[27] R. Koplatadze, "Oscillation criteria for higher order nonlinear functional differential equations with advanced argument," Nonlinear Oscillations, vol. 16, no. 1, pp. 44-64, 2013.

[28] R. Koplatadze, "On oscillatory properties of solutions of functional differential equations," Memoirs on Differential Equations and Mathematical Physics, vol. 3, pp. 3-179, 1994.

[29] M. K. Grammatikopulos, R. Koplatadze, and G. Kvinikadze, "Linear functional differential equations with Property A," Journal of Mathematical Analysis and Applications, vol. 284, no. 1, pp. 294-314, 2003. 


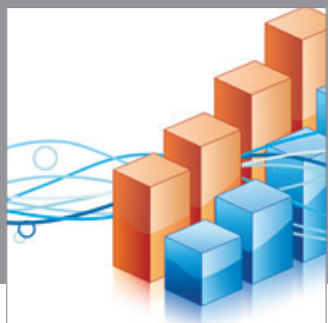

Advances in

Operations Research

mansans

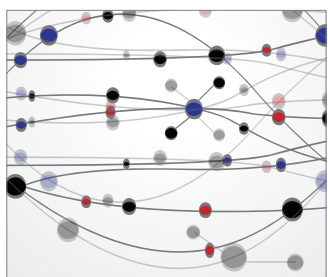

The Scientific World Journal
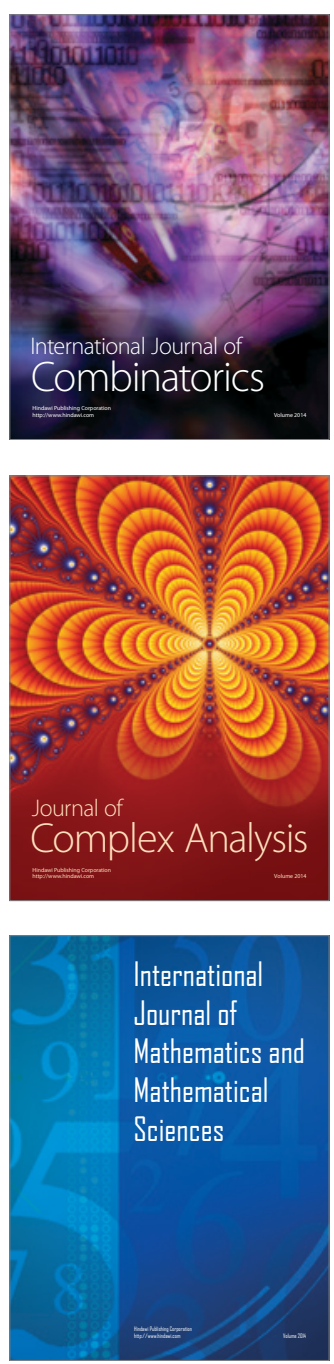
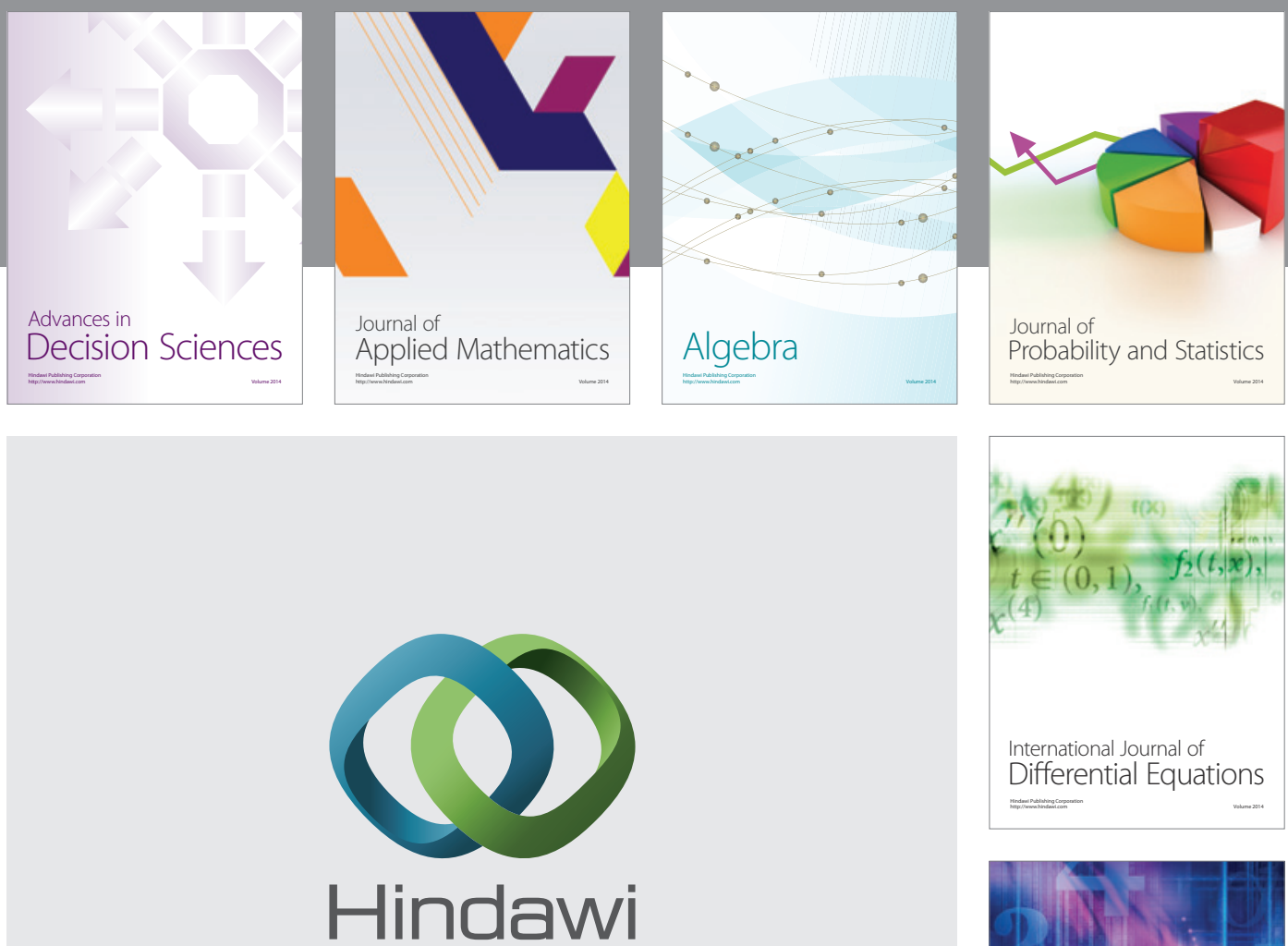

Submit your manuscripts at http://www.hindawi.com
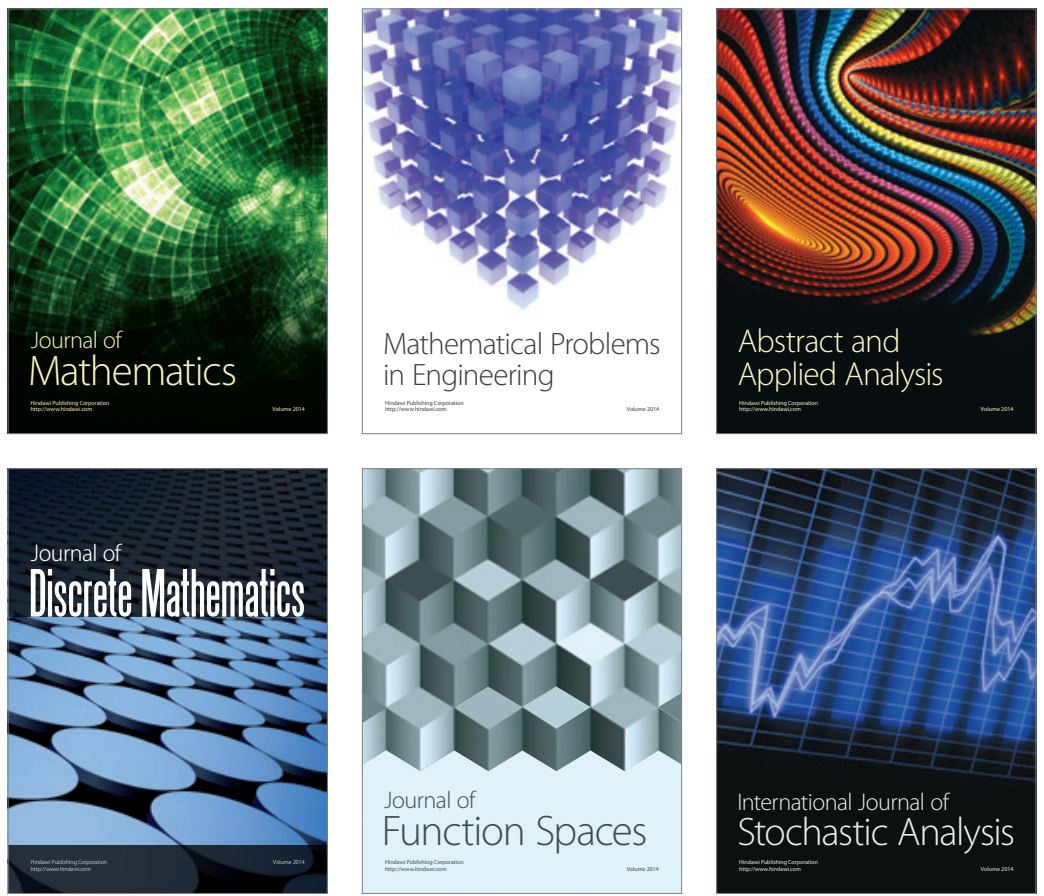

Journal of

Function Spaces

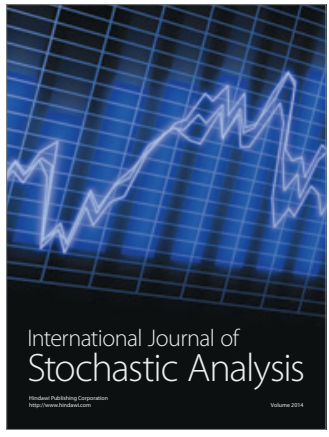

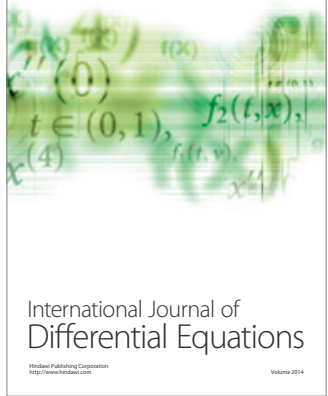
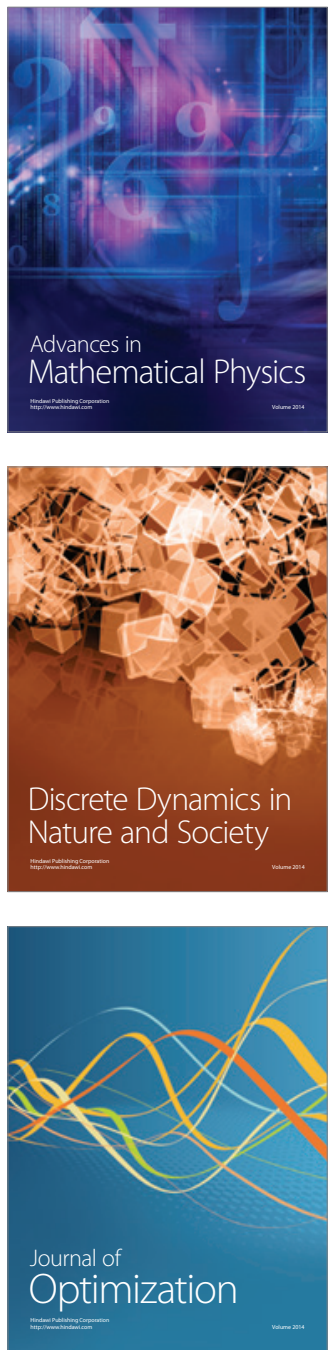